\section{P32 QUANTIFYING LEVELS OF PHYSICAL ACTIVITY IN PATIENTS WITH COPD: A US CROSS-SECTIONAL SURVEY}

${ }^{1} \mathrm{~B}$ Ding, ${ }^{2} \mathrm{D}$ Judge, ${ }^{2} \mathrm{M}$ Small, ${ }^{2} \mathrm{~N}$ Bent-Ennakhil, ${ }^{3} \mathrm{~S}$ Siddiqui. ${ }^{1}$ AstraZeneca Gothenburg, Mölndal, Sweden; ${ }^{2}$ Adelphi Real World, Macclesfield, UK; ${ }^{3}$ AstraZeneca, Gaithersburg, US

\subsection{6/thoraxjn-2017-210983.174}

Introduction and Objectives Physical inactivity can increase the burden of COPD and is a predictor of mortality and hospitalisations. There has been a paucity of data examining the relationship between physical activity and COPD treatment regimen. This study aimed to describe self-reported physical activity levels according to standard of care medication class (es) in COPD.

Methods Multicentre, cross-sectional, observational survey (Study: D5970R00003) conducted in the US. Patients $(\geq 40$ years) with a physician-confirmed diagnosis of COPD completed a questionnaire that included the Functional Performance Inventory-Short Form (FPI-SF) to assess physical activity (32 items/6 domains, rated from $1=$ much difficulty to $3=n o$ difficulty). Investigators provided information on treatment history.

Results 1775 patients participated (71.9\% Caucasian, 55.1\% male, $87.1 \%$ current/ex-smokers, mean age 65.2 years, mean BMI $27.5 \mathrm{~kg} / \mathrm{m}^{2}$ ). $14.8 \%$ of patients were classed as GOLD 2017 group A, $46.6 \%$ in group B, 2.6\% in group C and $36.0 \%$ in group D. Activity impairment based on FPI-SF scores was seen in patients across all treatment classes (Table), with the greatest impairment observed in patients receiving triple therapy and in the FPI-SF domains requiring most physical activity ('maintaining the household' and 'physical exercise').

Conclusions Patients with COPD who required triple therapy tended to report the lowest levels of physical activity. Longitudinal studies are needed to evaluate the effect of bronchodilator treatment on the relationship between lung function, COPD symptom burden and physical activity.

\section{P33 \\ WHAT MATTERS TO PEOPLE WITH COPD? OUTPUTS FROM WORKING TOGETHER FOR CHANGE}

F Early, M Lettis, JP Fuld. Cambridge University Hospitals NHS Foundation Trust, Cambridge, UK

\subsection{6/thoraxjnl-2017-210983.175}

Introduction and Objectives There is little qualitative research into what matters to people with COPD, in terms of managing their condition and supporting their wellbeing. We used a co-production methodology not previously applied in COPD, Working Together for Change (WTfC), to undertake a service evaluation. We aimed to identify what is important to people with COPD and what services would address these issues. Evaluation of the process has been described. ${ }^{1}$ Here we report the themes that emerged from WTfC that reflect what mattered to participants.

Methods WTfC began with 45 structured one-to-one discussions between an evaluator and participants identified from a GP list. We asked three questions: "What is working well in terms of your COPD care?", "What is not working well in terms of your COPD care?" and "What is important to you?" Responses were analysed using quality improvement methods in two co-production workshops involving COPD patients and carers (including some of the one-to-one participants), local health care professionals and voluntary sector organisations. Responses were themed by all participants working together with an expert facilitator. The group agreed a label for each theme and participants voted on the most important themes under the heading "What's not working."

Results The themes in each category are presented in Table 1. The six most important themes for "What's not working" were: "I don't think the right hand knows what the left hand is doing", "I can't get appointments when I want them", "I'm not treated as a person", "I can't do what I want to do", "I'm anxious and depressed" and "I can't eat well".

Abstract 32 Table 1 Physical activity by COPD treatment class

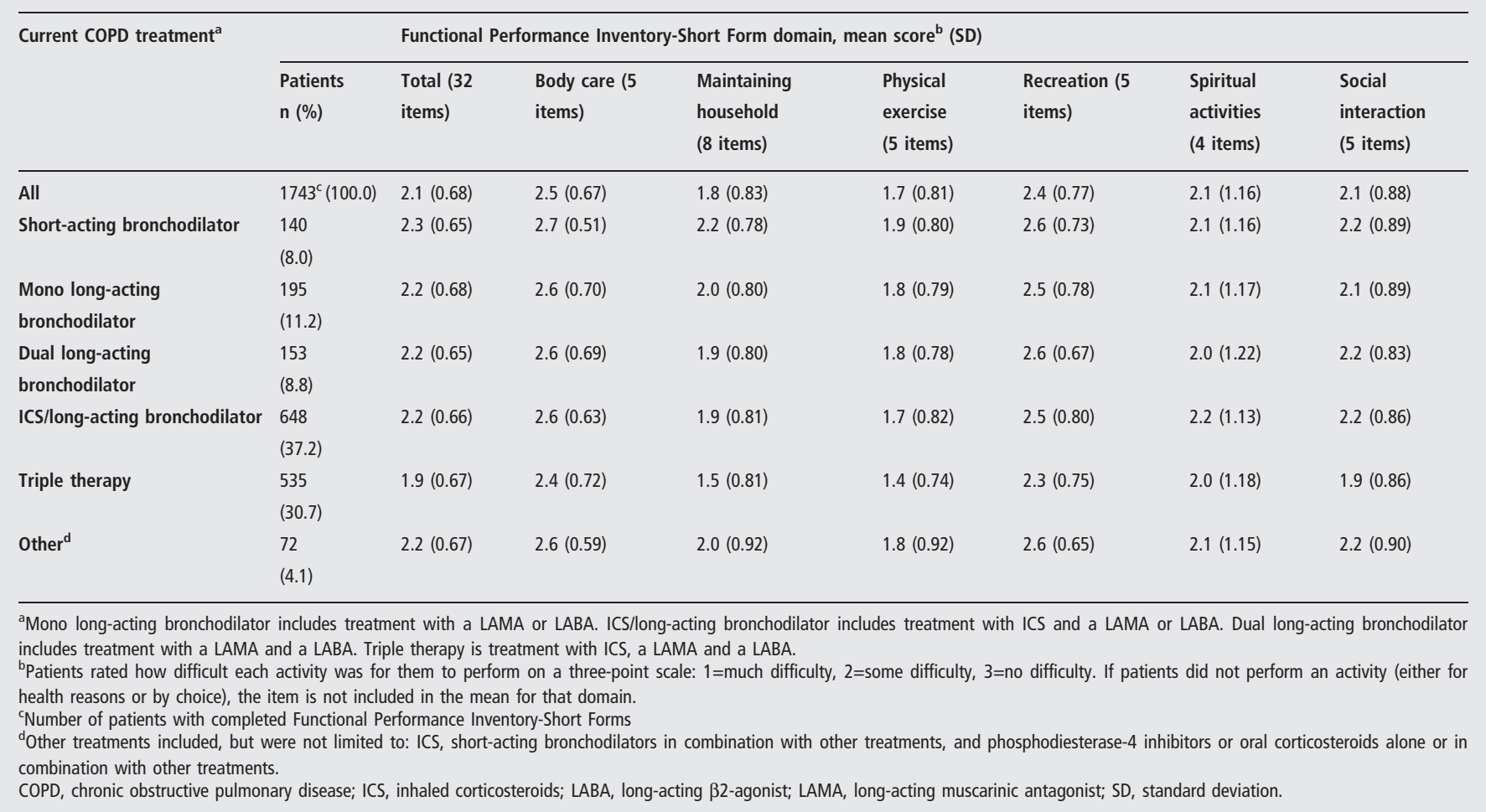

